\title{
ELEMENTOS RELEVANTES DEL PROYECTO DE UNA CENTRAL NUCLEAR
}

\author{
(RELEVANT TOPICS FOR THE DESIGN OF NUCLEAR POWER PLANTS)
}

Miguel Díaz-Llanos Ros, Ingeniero de Caminos Manuel Linares Moya, Ingeniero Aeronáutico Carlos Sánchez Ortiz, Supervisor de Diseño
Empresarios Agrupados, S. A. EPTISA, GHESA, TRSA

Magallanes, $3 \cdot 28015$ MADRID/ESPAÑA FORMAC-93

\section{RESUMEN}

Se presentan varios aspectos elegidos dentro de los caracteristicos del proyecto de una central nuclear. Se comparan los procesos de licencia en EE.UU. y en Alemania (RFA). Se introduce el programa de Garantia de Calidad como proceso esencial en este tipo de instalaciones.

Finalmente, se expone la integración de aplicaciones mecanizadas del proyecto, y se comentan cuatro áreas de desarrollo informático.

\section{SUMMARY}

Several topics were selected on the characteristics of Nuclear Power Plant design. The licensing process of USA and Germany (FRG) are compared. An introduction to the Quality Assurance Programme as an essential item in the nuclear field is presented.

The integration of computer applications as part of the engineering approach to design is explained and comments are made on four computer developments.

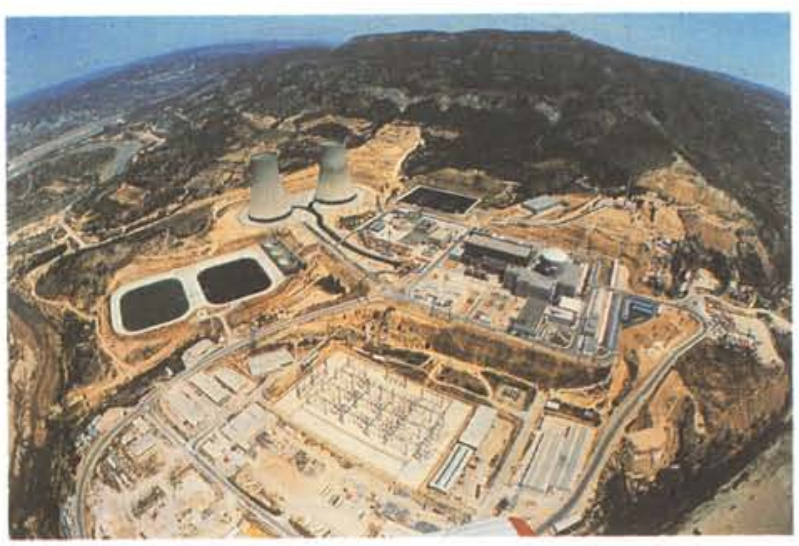

Vista general de una central nuclear.

\section{INTRODUCCIÓN}

Las centrales nucleares a efectos de proyecto y construcción representan un gran desafio por parte de las organizaciones que se encuentran involucradas en su realización.

(c) Consejo Superior de Investigaciones Científicas Licencia Creative Commons 3.0 España (by-nc)
Se presentan de forma somera algunos temas especificos del proyecto de este tipo de realizaciones tales como: Organización y estructura del proyecto, Licencia, Garantia de Calidad y Mecanización.

\section{ORGANIZACIÓN Y ESTRUCTURA DE PROYECTOS}

La realización de proyectos de Centrales Nucleares conlleva una dependencia jerárquica por áreas de especialización: civil, mecánica, eléctrica, etc. (ver gráfi$\cos 1,2$ y 3 ). Con una dependencia funcional por proyectos a esta organización, con doble dependencia, se le denomina matricial.

Este tipo de organización, típica de las empresas de ingenieria que desarrollan varios proyectos al mismo tiempo, permite obtener una mejor utilización de los recursos humanos existentes y una más rápida transferencia de experiencias tecnológicas entre proyectos, lográndose así una mayor unificación en cuanto a criterios de actuación.

http://informesdelaconstruccion.revistas.csic.es 

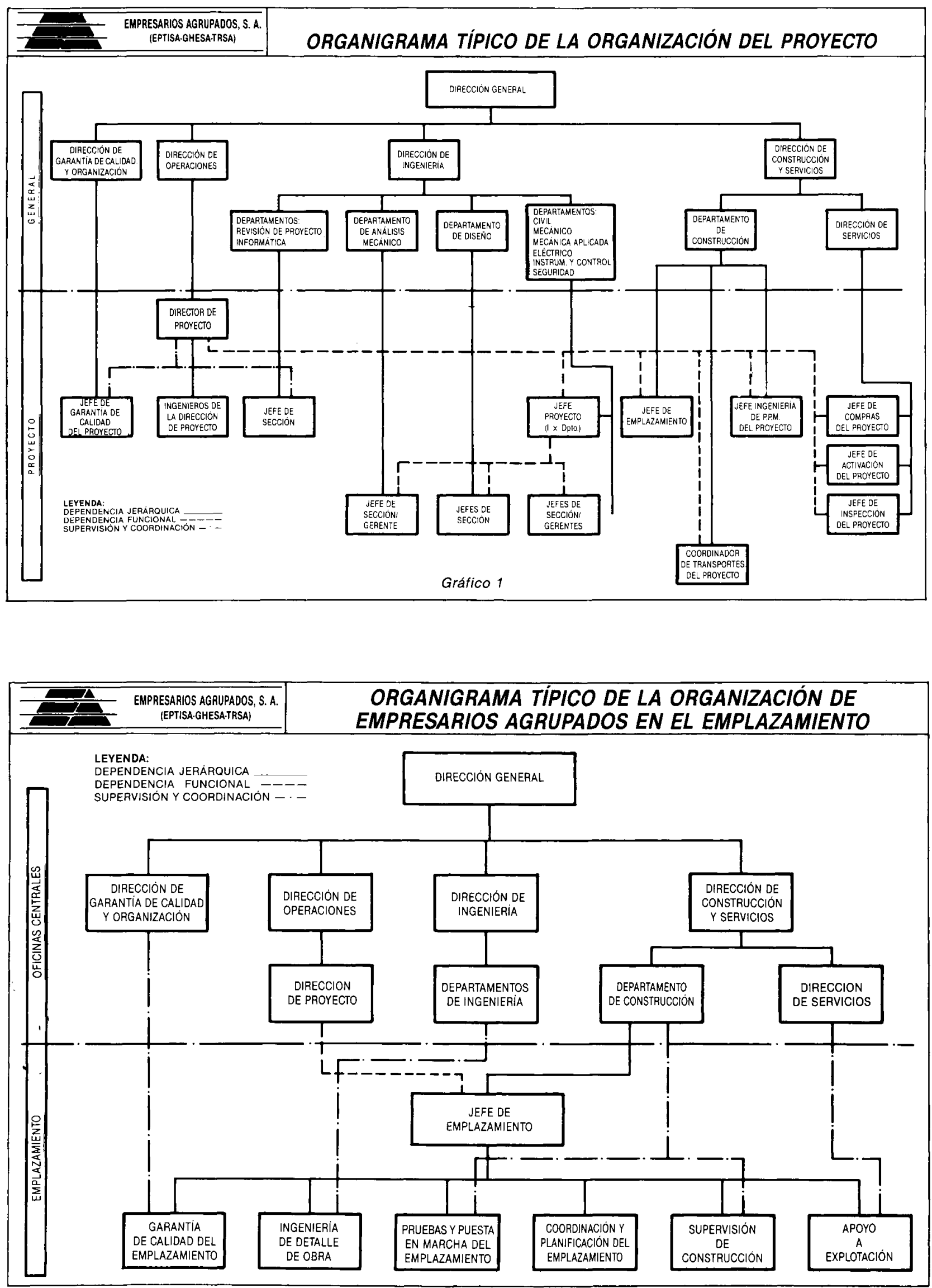

Gráfico 2 


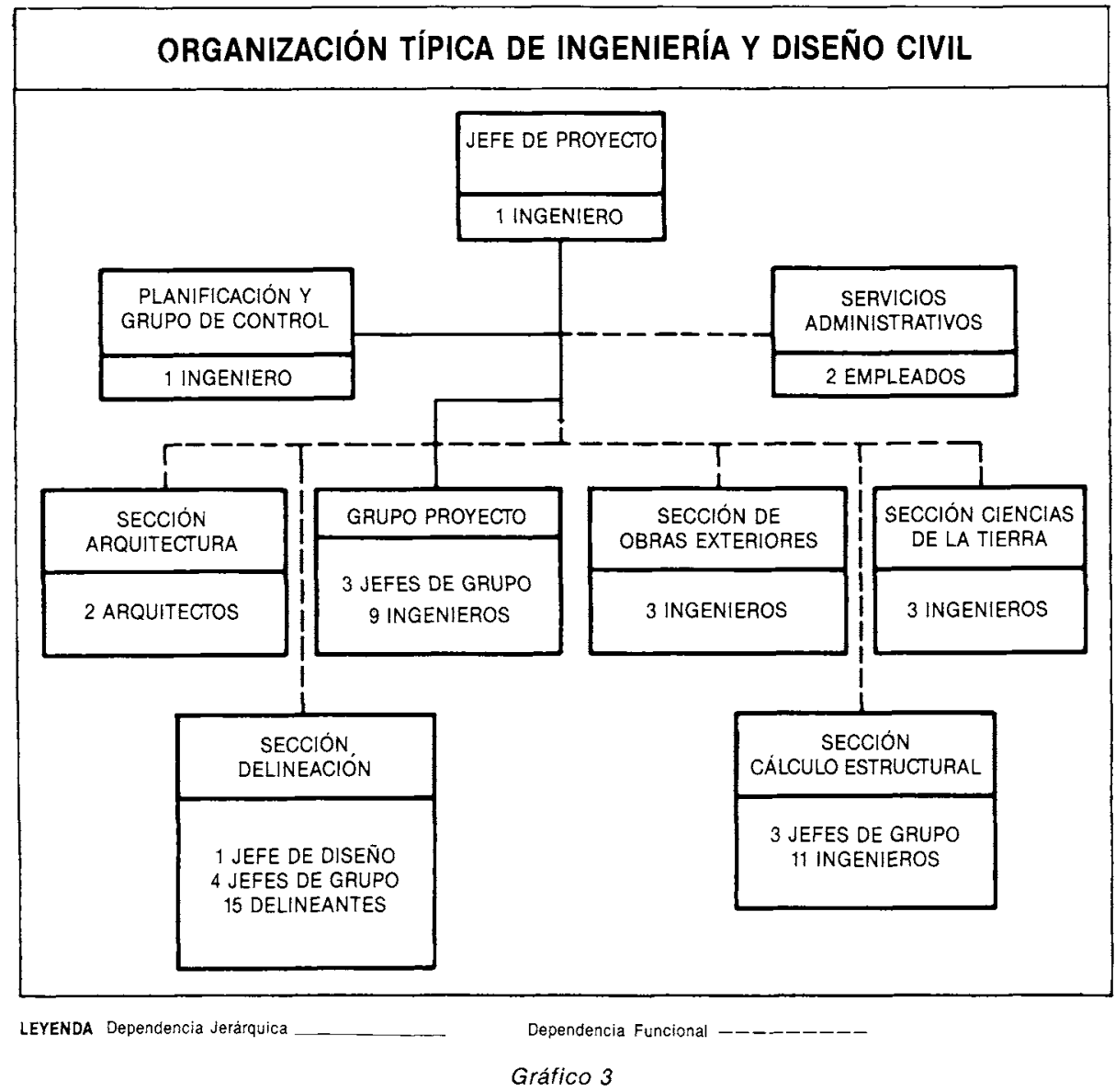

\section{LICENCIA}

Los requisitos reguladores que deben cumplirse en el desarrollo de una Central Nuclear, se pueden clasificar en los siguientes tipos:

a) Requisitos legales obligatorios por Ley, Decreto, Orden, Reglamento, etc., de la legislación española vigente que es aplicable genéricamente a este tipo de proyectos.

b) Tratados y convenios internacionales a los que está adherido el Estado Español.

c) Requisitos legales específicos para cada proyecto, establecidos explícitamente en las autorizaciones previas y de construcción, asi como en el Permiso de Explotación Provisional, incluyendo aquella le. gislación obligatoria, vigente y aplicable del país de origen del Reactor en aquellas áreas no cubiertas por la legislación española.

Incluimos una breve descripción de los procesos de licencia en EE.UU. y RFA como referencia, dado que suele ser normal seguir alguno como modelo para países donde no se ha desarrollado una tecnología específica.

\section{Proceso de licencia en EE.UU.}

El proceso de licencia en EE.UU. de Norteamérica consta de las siguientes fases:

a) Permiso de Construcción. Consta de las siguientes etapas:

- El propietario presenta a la Nuclear Regulatory Commission (NRC) el Estudio Preliminar de Seguridad (EPS).

- La NRC realiza una revisión previa del EPS de acuerdo con la Guía Reguladora 1.70 "Standard Format and Content of Safety Analysis Reports for Nuclear Power Plants, Light Water Reactor (LWR) Edition", posteriormente realiza una revisión profunda de acuerdo con el NUREG 0800 "Standard Review PIan (SPR)" y emite un informe de la 
revisión indicando sus conclusiones, aspectos pendientes y compromisos a cumplir por el pro. pietario.

- Se abre un período de información pública, para presentar las alegaciones que se consideren oportunas.

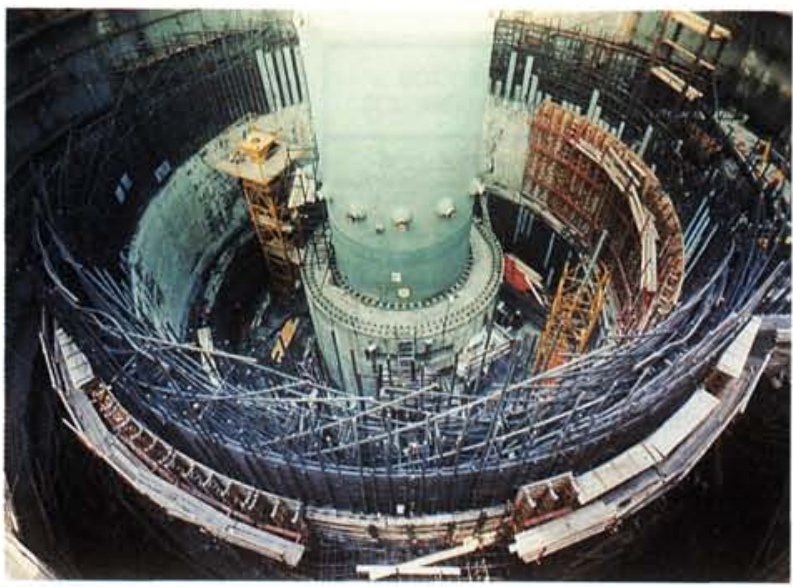

Edificio del reactor de una central tipo BWR.

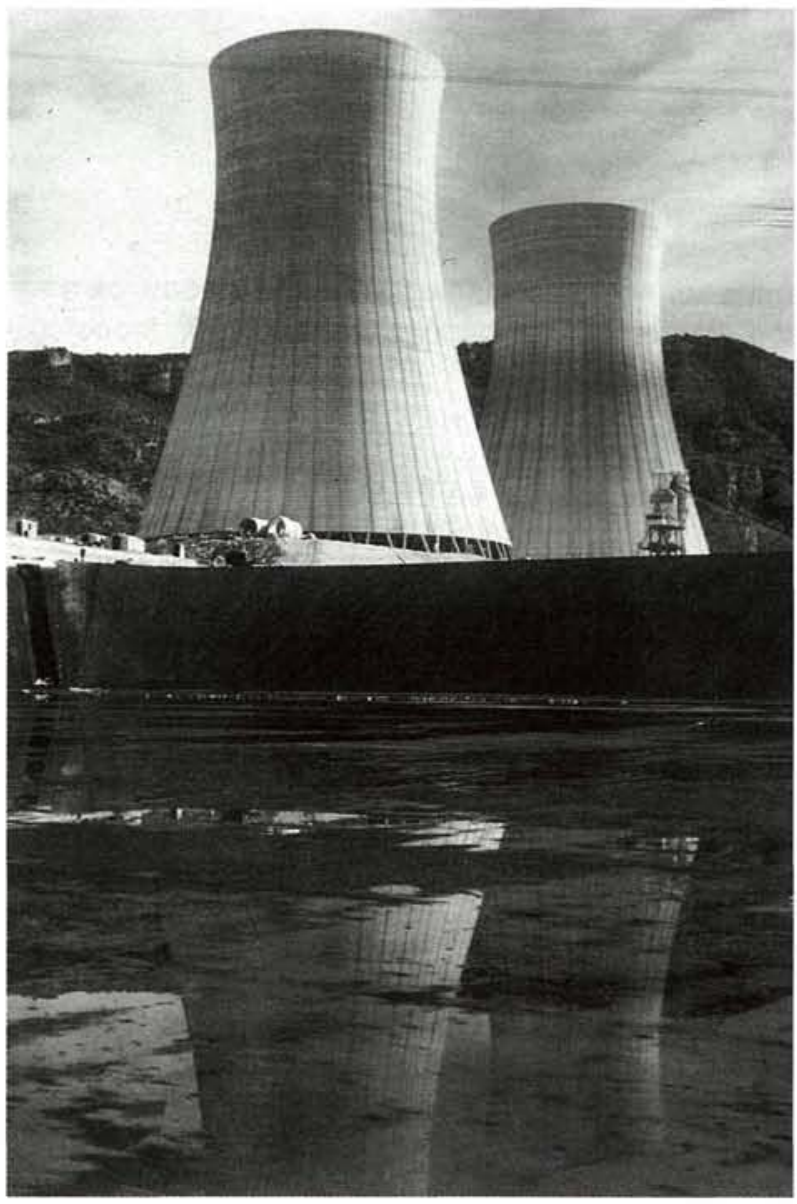

Torres de refrigeración.

(C) Consejo Superior de Investigaciones Científicas Licencia Creative Commons 3.0 España (by-nc)
- El Advisory Committee Reactor Safety (ACRS) realiza una revisión independiente del EPS presentando un informe a la NRC sobre los resultados de la evaluación así como su recomendación so. bre la concesión o no del permiso.

- La NRC, sobre la base de su informe, el de la ACRS y las alegaciones presentadas decide sobre la concesión o no del permiso de construcción.

b) Revisión del Impacto Ambiental. Consta de las siguientes etapas:

- El propietario presenta a la NRC un Informe Am. biental.

La NRC realiza una revisión de este informe de acuerdo con la Guia Reguladora 4.2 "Preparation of Environmental Reports for Nuclear Power Plants"; además hace un análisis del medio am. biente como consecuencia de la construcción y operación de la Central y lo distribuye para comentarios a las agencias federal, estatal y locales.

Finalmente lo publica incorporando los comentarios que estimen oportunos.

c) Revisión Antimonopolio.

- La NRC y el Fiscal General realizan esta revisión, para ver si se cumple la legislación antimonopo. lio existente.

\section{d) Licencia de Operación.}

- Se vuelven a repetir las etapas incluidas dentro de la fase de permiso de construcción.

Independientemente de estas cuatro fases y una vez que la central está en operación la NRC realiza vigilancias e inspecciones periódicas, pudiendo solicitar modificaciones en los elementos como consecuencia de las exigencias impuestas por nuevos requisitos. El propietario debe informarle de los sucesos que supongan una desviación de la operación prevista.

\section{Proceso de licencia en RFA}

El proceso de licencia en la República Federal de Alemania (RFA) consta de las siguientes fases:

- Primera licencia parcial. Autorizando la localización del emplazamiento, las características básicas de la planta, las estructuras subterráneas de hormigón y la construcción parcial de los edificios auxiliar y del reactor.

http://informesdelaconstruccion.revistas.csic.es 
- Segunda licencia parcial. Autorizando la terminación de la construcción de los distintos edificios.

- Tercera licencia parcial. Autorizando la instalación de diversos sistemas mecánicos y eléctricos.

- Cuarta licencia parcial. Autorizando el transporte y manipulación de los conjuntos combustibles y la realización de pruebas prenucleares y nucleares.

- Quinta licencia parcial. Autorizando la realización de pruebas nucleares de potencia y la manipulación de combustible y demás sustancias readiactivas.

- Licencia para la utilización de agua. Autorizando la toma de agua para refrigeración y su desagüe, una vez tratada.

Las autoridades de cada uno de los once estados federales de la RFA son los responsables de la concesión de permisos y licencias. Para realizar esta actividad solicitan al Technischer Uberwachungs-Verein (TüV) y al Gesellschaft fur Reaktorsicherheit (GRS) que analicen la documentación en relación con el cumplimien. to de legislación.

\section{Comparación de procesos}

El proceso de licencia utilizado en la RFA es conceptualmente diferente al que se utiliza en EE.UU. de Norteamérica o en España, no sólo en cuanto a organización sino también en su filosofía.

La filosofía utilizada en la RFA se basa en la revisión y aprobación de toda la documentación generada en el diseño, construcción y puesta en marcha, tal como planos, especificaciones, procedimientos, informes de pruebas, etc., mientras que la filosofia utilizada en EE.UU. de Norteamérica o en España se basa en la presentación y aprobación de una documentación de licencia cuyo contenido está previamente definido.

\section{GARANTIAA DE CALIDAD}

En las instalaciones nucleares adquiere una especial relevancia el concepto diferenciado de garantía de calidad frente a la especificación de calidad y control.

\section{Generalidades}

El término "garantía de calidad" se define como "el conjunto de acciones planificadas y sistemáticas necesarias para proporcionar una confianza adecuada en que una estructura, sistemas y componentes se comportan adecuadamente en servicio". En el campo de las instalaciones nucleares, estas estructuras, sistemas y componentes son los que previenen o mitigan las consecuencias de accidentes postulados que pudieran causar un riesgo indebido a la salud y seguridad del público.

Los criterios de planificación de estas acciones vienen determinados en las siguientes normativas:

- UNE 73-401 "Garantía de calidad en centrales nuclea. res".

- USNRC 10 CFR50, Ap, B "Quality Assurance Criteria for Nuclear Power Plants and Fuel Reprocessing Plants".

- OIEA 50-C-QA "Garantia de calidad para la seguridad en las centrales nucleares".

Los requisitos que imponen la garantia de calidad son de aplicación a todas las actividades que afectan a las funciones de seguridad tales como: el diseño, la compra, la fabricación, el manejo, el transporte, el almacenamiento, la limpieza, el montaje, la instalación, las pruebas, la operación, el mantenimiento, la reparación, la recarga, y las modificaciones.

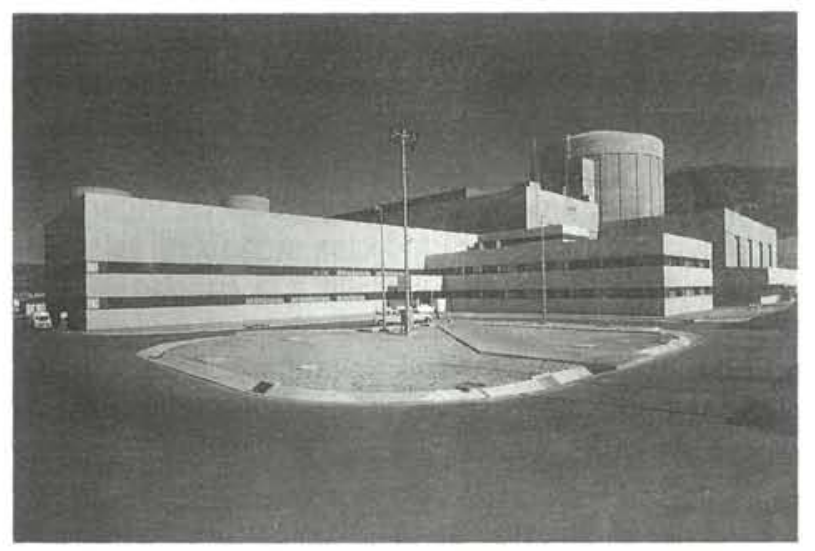

Vista parcial de una central nuclear.

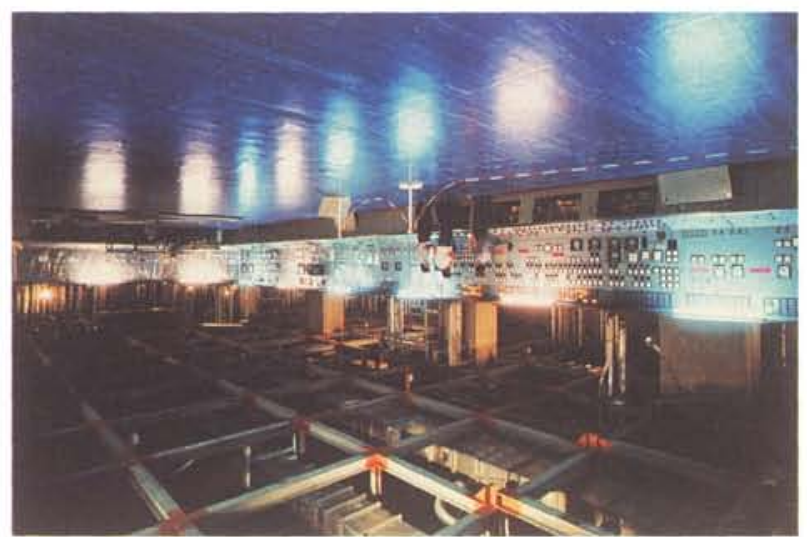

Sala de control durante la fase de montaje. 
Estas actividades incluyen tanto las funciones de conseguir los objetivos de calidad, como las funciones de garantía de calidad. Estas segundas son las que aseguran que se implante un Programa de Garantía de $\mathrm{Ca}$ lidad efectivo, por medio de comprobaciones, auditorias e inspecciones.

\section{Programa General de Garantia de Calidad}

De acuerdo con la normativa citada en el apartado anterior, el solicitante o propietario de una instalación nuclear debe establecer un Programa de Garantía de Calidad y ejecutarlo durante toda la vida útil de la instalación.

La dirección de la Garantía de Calidad debe ser una unidad independiente de las restantes direcciones con misiones de producción, cuya responsabilidad es supervisar y controlar la implantación de la política de calidad establecida.

De manera especifica, tiene encomendadas las siguientes funciones:

a) Desarrollar la política de Garantía de Calidad establecida por el Director General, si asi se decide, de acuerdo con el Propietario, asegurando que se ejecuta correctamente.

b) Aprobar el Programa de Garantía de Calidad (PGC) y sus documentos complementarios (Lista de Requisitos Reguladores y "Clasificación de Calidad").

c) Verificar los procedimientos y guias de ingenieria y diseño de todas las unidades organizativas relativos a actividades que afecten a la calidad.

d) Establecer los programas de auditorias y controles necesarios para asegurar el cumplimiento de los requisitos definidos en el PGC.

e) Informar al Director General del estado de implantación del PGC, asi como del desarrollo de las audi. torías.

f) Establecer programas de formación y enseñanza del personal que proporcione los niveles de cualificación requeridos.

\section{MECANIZACIÓN DE LOS PROYECTOS}

Entre las actividades que componen un Proyecto nuclear, se pueden identificar cuatro grupos de aplicaciones informáticas:

- Los cálculos técnicos de los edificios, sistemas y componentes.
- Las aplicaciones requeridas por la gestión del pro. yecto.

- El diseño asistido con ordenador (CAD y GIS).

- Las aplicaciones orientadas a la integración de actividades.

Unas y otras presentan caracteristicas y tienen requisitos peculiares que conviene analizar separadamente.

\section{CÁLCULOS TÉCNICOS}

Pertenecen a este grupo los programas de análisis estructural o de sistemas de tuberías, de cálculos de dosis, de recorrido de cables, etc. Suelen tener entrada de datos secuencial, como salida de listados de formato tabular, y se ejecutan por lotes (batch). Su interactividad es escasa, salvo en la entrada de datos ayudada, a veces, por funciones de edición.

Los programas que resuelven estos cálculos son los de origen más antiguo de los utilizados actualmente. En la figura 1 se observa el modelo axilsimétrico de una contención de hormigón que utilizando el método de elementos finitos permiten analizar y predecir el com. portamiento no lineal, fisuración del hormigón y de la estructura bajo cargas extremas o de accidente. En otros casos es necesario comprobar el dimensionamiento de estructuras de acero con geometrías complejas pero asimilables a un estado de deformación plana (Fig. 2).

Existen circunstancias donde una misma estructura es analizada sometida a acciones mecánicas (inclusive sísmica) asi como térmicas, en las hipótesis de com. binación de los distintos códigos de hormigón. En la figura 3 se pueden observar los resultados del modelo tridimensional de una piscina de combustible compro-

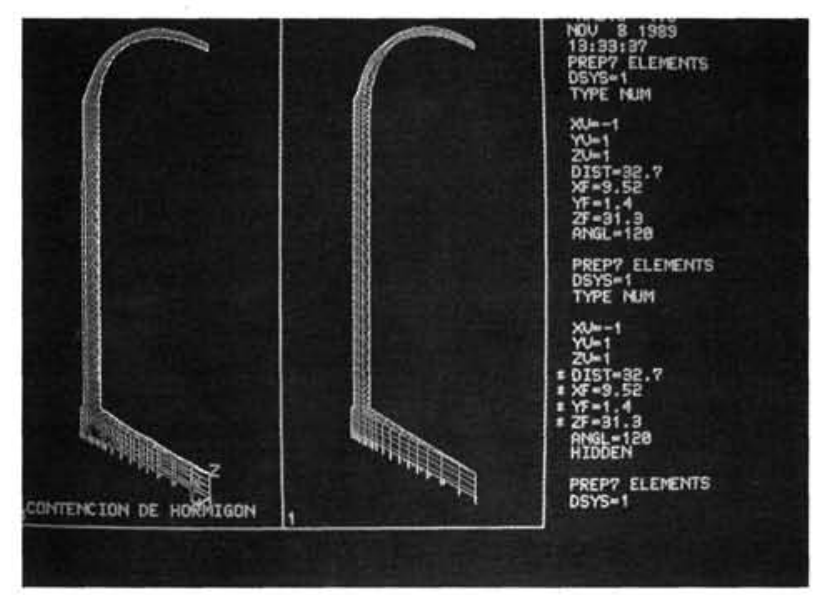

Fig. 1.-Modelo axilsimétrico de análisis no lineal de una contención de hormigón armado.

http://informesdelaconstruccion.revistas.csic.es 


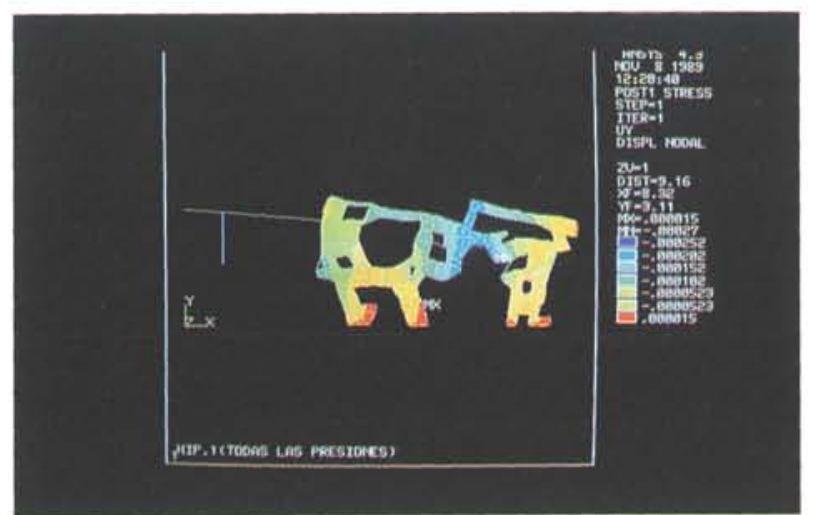

Fig. 2.-Resultados de tensiones en una estructura metálica modelizada por elementos finitos.

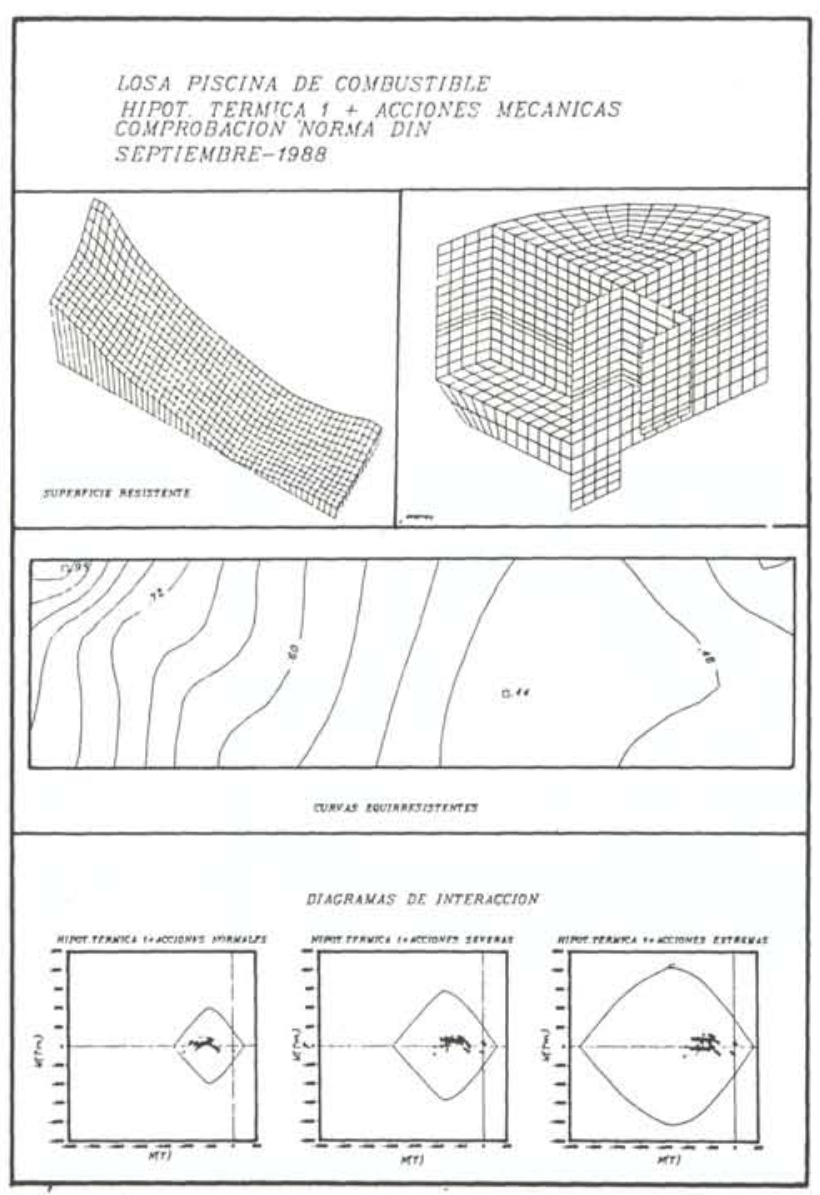

Fig. 3.-Modelización de la piscina de combustible y verificación de resultados con diagrama de interacción bajo distintos códigos de hormigón (EH, ACI, DIN).

bada según la normativa alemana DIN 1045 en acciones normales, acciones sísmicas de parada segura y sismo de diseño.

En estos tres ejemplos mostrados en las figuras 1, 2 y 3 se puede apreciar la ventaja de una salida gráfica donde se visualiza de forma rápida los elementos más tensionados, frente a los primitivos listados de ordenador.

(C) Consejo Superior de Investigaciones Científicas Licencia Creative Commons 3.0 España (by-nc)
La evolución de los programas, junto con la larga du. ración de los proyectos nucleares, hacen que, a lo largo de un proyecto, coexistan varias versiones del mismo programa que se han ido utilizando a medida que iban estando disponibles nuevas mejoras 0 , contrariamente, se siga utilizando una versión ya antigua para mantener la coherencia de la normativa utilizada a lo largo de todo el proyecto. Esta circunstancia obliga a llevar un minucioso control de las versiones utilizadas en cada uno de los cálculos del Proyecto y de su documentación.

\section{GESTIÓN DEL PROYECTO}

Estos programas han sufrido una enorme evolución en los últimos años. Han pasado de ser meros listados de documentos a ser auténticos sistemas de Información del Proyecto (SIP).

Desde el punto de vista del usuario final, el SIP es una herramienta que proporciona información procesada en la que se pueden basar decisiones relacionadas con la gestión.

En la actualidad se tiende a organizar los SIP en forma de sistemas de bases de datos capaz de cubrir la totalidad de las actividades de gestión.

\section{LOS SISTEMAS DE DISEÑO ASISTIDO CON ORDENADOR (CADIGIS)}

Cada día la automatización adquiere mayor importancia y actualmente se han desarrollado fundamentalmente dos sistemas de tratamiento gráfico por ordenador que son:

\section{Los sistemas de CAD (Computer-aided Drafting and} Design).

2. Los sistemas de GIS (Geography Information System).

Normalmente ambos tipos de sistemas se confunden como si fuesen iguales pero técnicamente son muy diferentes.

Los sistemas CAD nacieron como meros tecnígrafos electrónicos dotados de aptitudes singulares: perfección en el trazado de líneas, manejo de transformaciones geométricas (traslación, giro, simetría, semejanza, transformaciones proyectivas, etc.), inserción de figuras típicas, repetición de partes completas de un dibujo, cálculos ligados a la geometria de las figuras, acotación automática, etcétera. 
Posteriormente, se incorporan atributos no gráficos a los sistemas de CAD almacenándose separadamente como datos tabulares que pueden ser interrogados $\mathrm{V}$ usados para manipular la salida gráfica.

De esta forma se observa una evolución de los sistemas de CAD que pasan de ser solamente una herramienta de diseño y dibujo a un propósito más amplio de gestión de datos.

Una de las primeras metas que surgen cuando se analizan planos o croquis es extraer cierta información contenida en el dibujo. Puede tratarse de la lista de equipos, o las coordenadas de los nudos de una red de drenaje contenida en el plano.

Una peculiaridad de muchos sistemas CAD, es la capacidad para asignar la información gráfica o numérica de un determinado plano a un nivel de información elegido por el usuario. Cada nivel de información actúa como una hoja transparente que contiene solamente la información asignada a dicho nivel. (Figs. 3, 4, 5, 6 y 7 ).

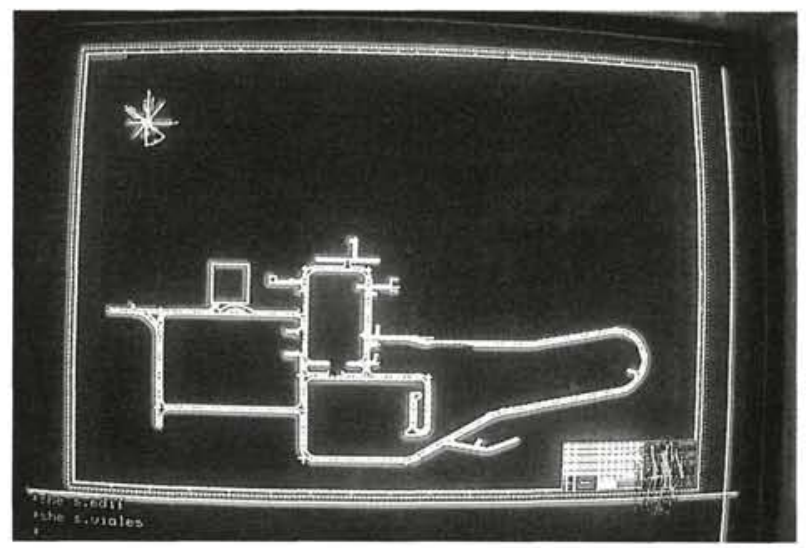

Fig. 4.-Viales.

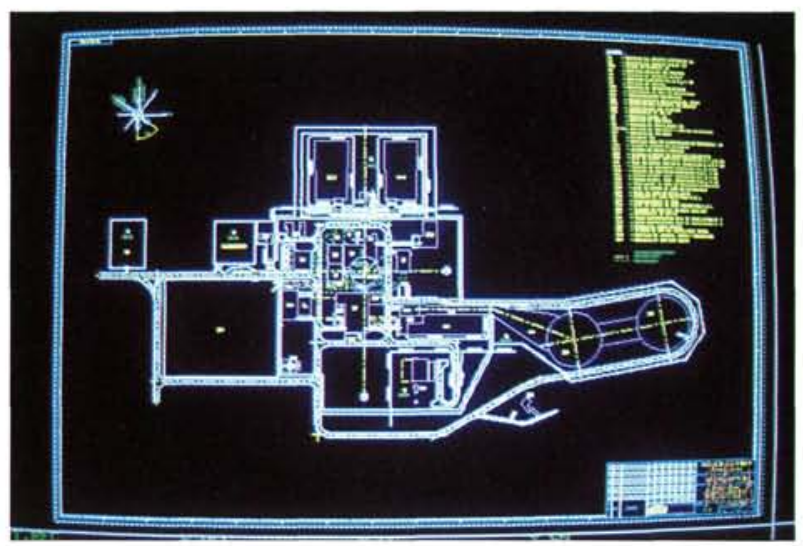

Fig. 5.-Viales $y$ edificios.

(C) Consejo Superior de Investigaciones Científicas Licencia Creative Commons 3.0 España (by-nc)
A principios de los años sesenta se empieza a desa. rrollar un concepto de software conocido como GIS (Geographic Information System) el cual se concibe para gestionar grandes volúmenes de información sobre el medio natural y el entorno geográfico.

En un sistema GIS los datos espaciales no se almacenan como gráficos sencillos o símbolos, sino de una forma analítica independiente de las representaciones específicas, generándose automáticamente una relación topológica que define los elementos espaciales con los que va a operar un GIS, y que son: puntos, lineas y polígonos. Además, esta relación topológica une intimamente estos elementos (puntos, líneas y polígonos) con datos tabulares (atributos) almacenados en una base de datos. De esta forma con una organización GIS se obtiene una mayor simplicidad en el uso y análisis de datos geográficos ya que sólo se recurre a las coordenadas cartográficas a la hora de crear salidas gráficas.

Un GIS no debe ser utilizado sólo como una herramienta de diseño y dibujo, pues además de almacenar y re-

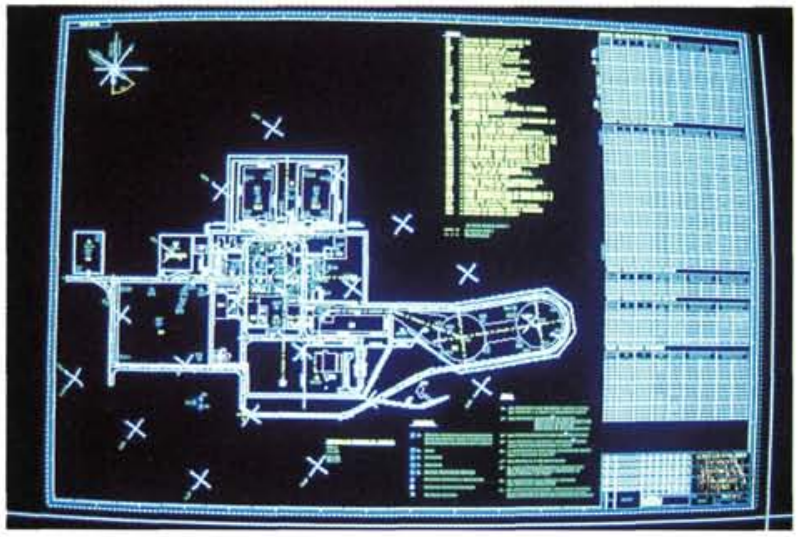

Fig. 6.-Planta general.

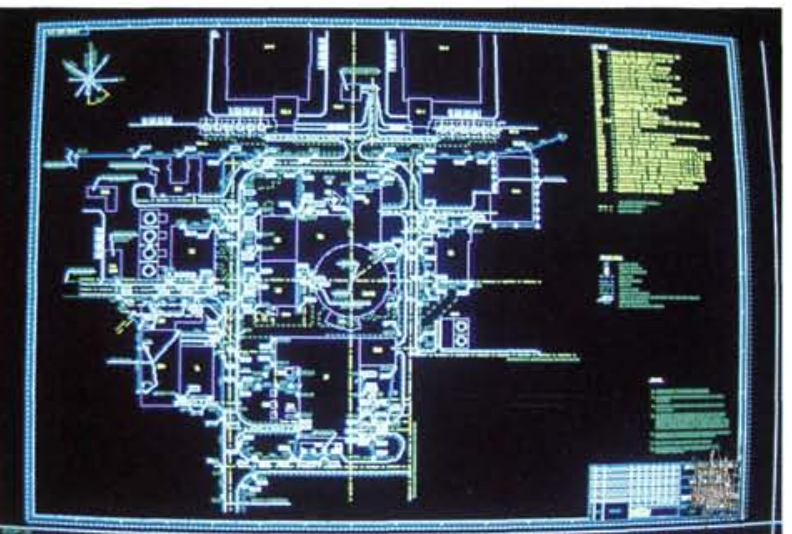

Fig. 7.-Red de drenaje de pluviales (ZOOM).

http://informesdelaconstruccion.revistas.csic.es 


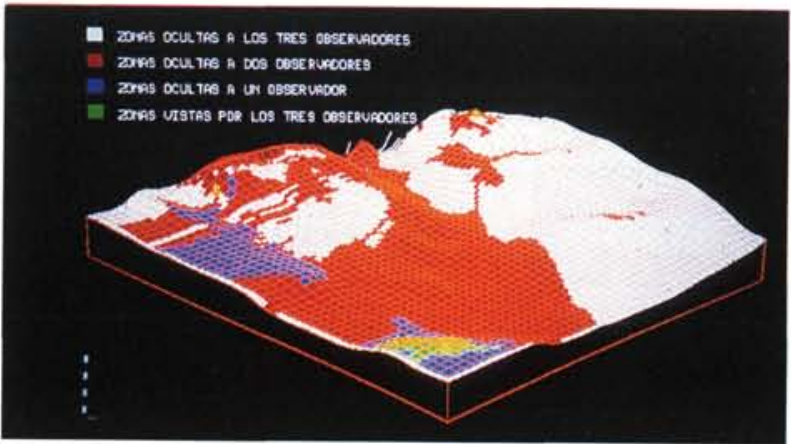

Fig. 8.-Tipología de zonas de visión $3 D$.

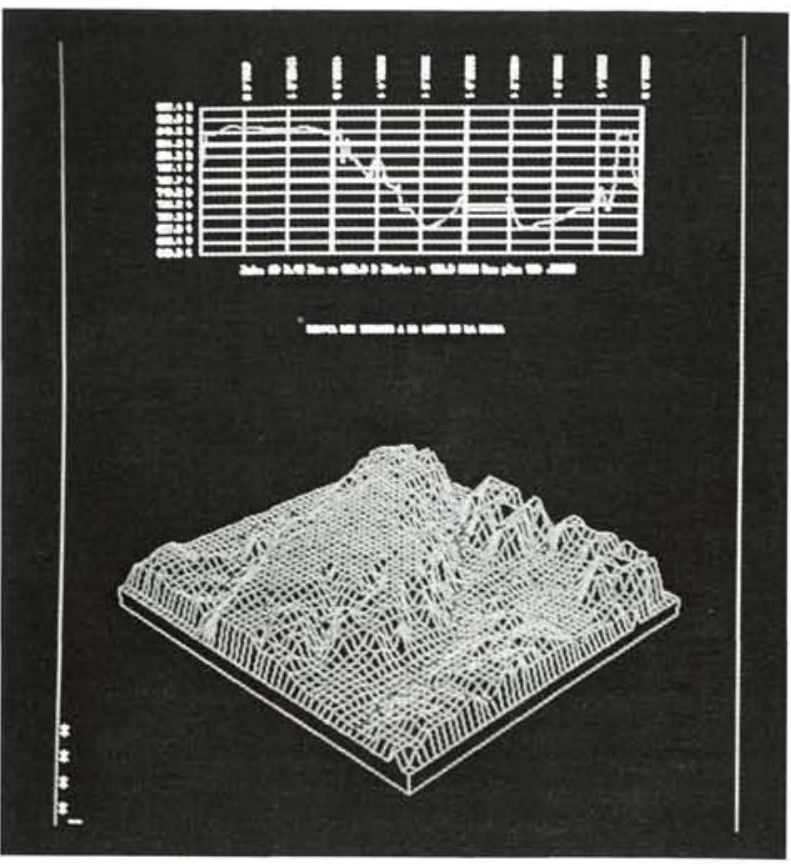

Fig. 9.-Perfil topográfico $3 D$.

generar gráficos, genera éstos basándose en la información relacional que contiene su base de datos. Esta información puede utilizarse con una gran flexibilidad, cualidad que es especialmente interesante cuando se emplea la misma información cartográfica con diferentes propósitos. Por ejemplo, en un Ayuntamiento se puede desear obtener mapas que diferencien las parcelas según su distinto uso, o según su distinta carga impositiva, el nombre del propietario, etc.; este sistema de información permite automatizar el formato de salida, sombreados y simbología empleada según los distintos propósitos que se consideren.

Más allá de esta flexibilidad gráfica, esta nueva tecnologia de software facilita la gestión, organización, análisis, pregunta y salida gráfica de grandes volúmenes de información espacial. Además introduce herramientas de análisis y manipulación como la superposición e intersección de mapas, análisis de proximidad, análisis de redes, ..., cuestiones que hacen de este sistema una magnífica herramienta para la gestión de bases de datos territoriales. (Figs. 8, 9, 10, 11 y 12).

(c) Consejo Superior de Investigaciones Científicas Licencia Creative Commons 3.0 España (by-nc)

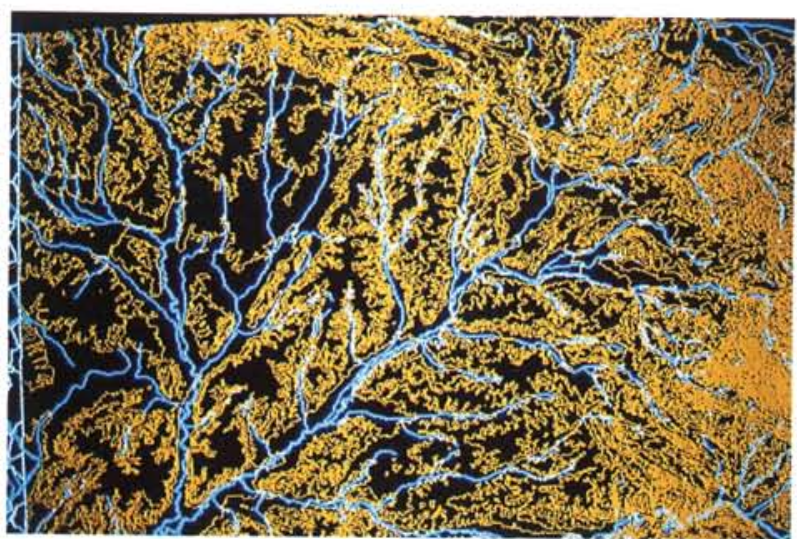

Fig. 10.-Topografia de zona.
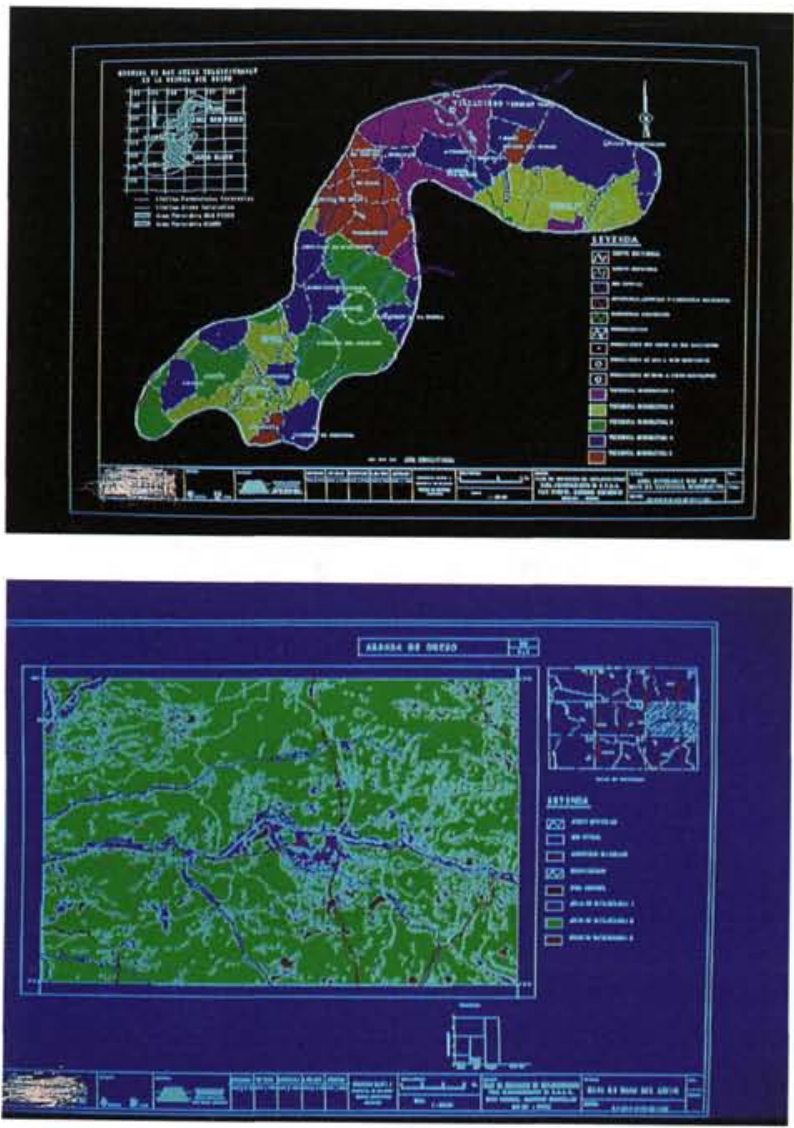

Figs. 11 y 12.-Mapas con datos territoriales de zonas.

\section{Desarrollos especificos}

En la actualidad se dispone de aplicaciones especificas que aportan un procedimiento estructurado para, aprovechando las capacidades de los sistemas, y dentro del contexto general de los distintos paquetes gráficos, diseñan aquellos elementos que configuran una estructura, obteniendo los planos de construcción, visualizaciones y banco de datos necesarios. (Figs. 13, 14 y 15). 


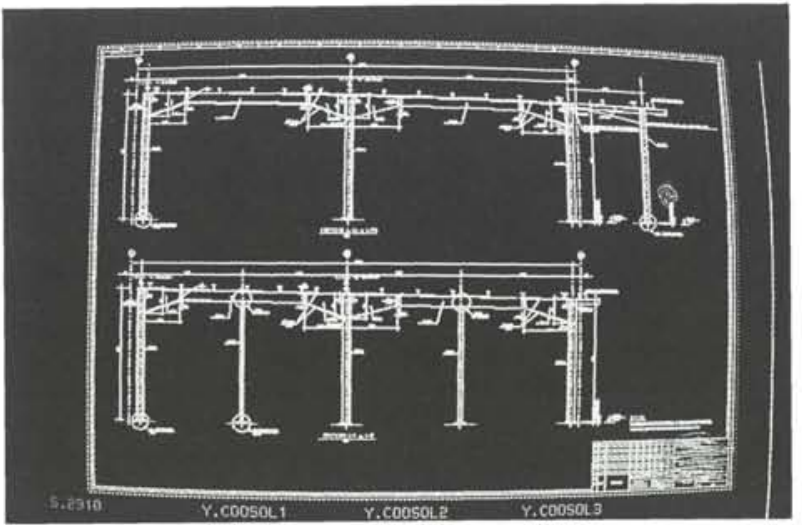

Fig. 13.-Pórticos metálicos.

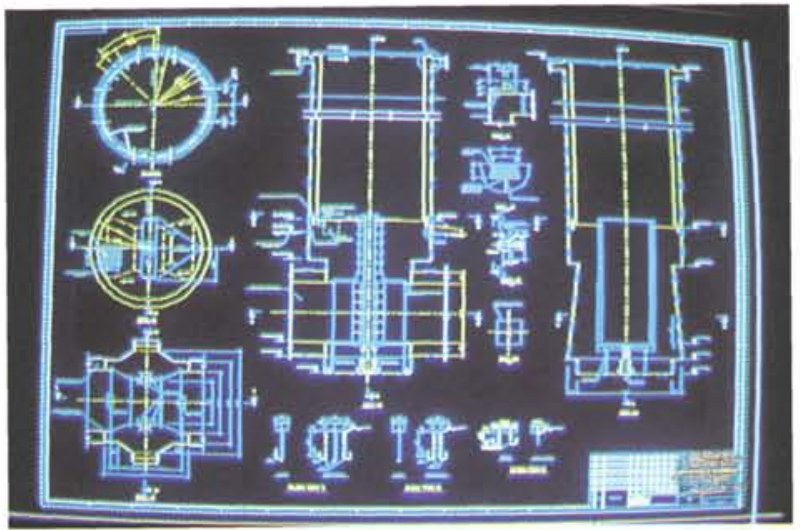

Fig. 14.-Pozo de compuerta.

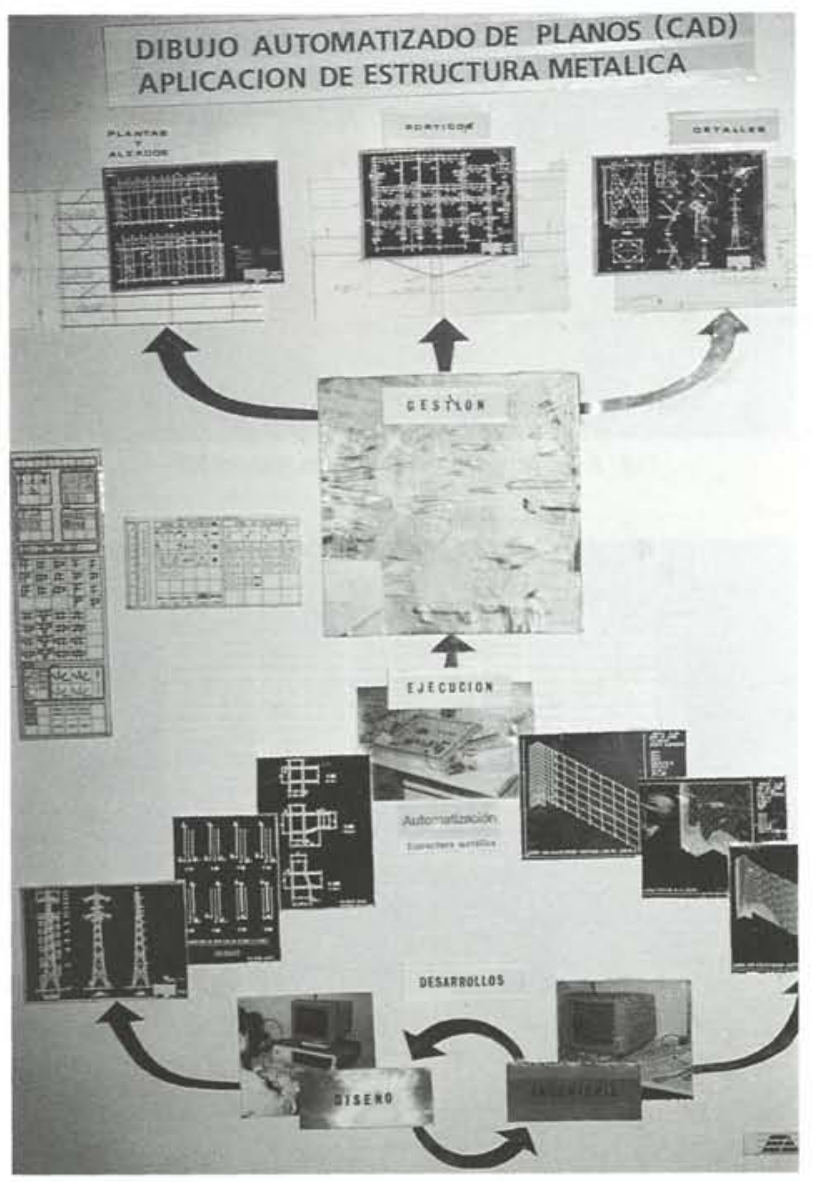

Fig. 15.-Aplicación especifica de estructura metálica. (Flujo de procesos).

\section{INTEGRACIÓN DE APLICACIONES}

En la realización de los Proyectos, la integración aspira a cubrir la Ingeniería, el Diseño y la Gestión.

Si se tiene un programa que calcula tuberías y otro que calcula soportes es racional enlazarlos de modo que la situación y rigidez exigida a los soportes sean datos directamente transmitidos para el análisis de las tuberias. Similarmente, las reacciones obtenidas de dicho cálculo entrarán como datos en el cálculo de los soportes. Si a ello se añaden unas capacidades gráficas que dibujen los isométricos y los soportes y se listan los materiales requeridos para cada uno de ellos se tendrá un esquema simplificado de lo que cabe esperar de un paquete integrado de aplicación para tu. berias.

Dentro del diseño de la planta se consideran los diferentes tipos de documentos producidos: Planos, esque. mas, cálculos, listas y otros documentos (especificaciones, procedimientos de inspección, etc.) con vistas a lograr una interacción completa entre todos ellos.

No parece que, actualmente, se pueda informatizar el proyecto de una Central Nuclear completa, con el grado de automatismo que se imagina ya como posible, debido al tamaño del problema, pero no cabe duda de que la evolución va en este sentido. 